MEXАНIKA

MECHANICS

https://doi.org/10.15407/dopovidi2021.06.032

удК 539.3

О. Ю. Чирков, https://orcid.org/0000-0003-1916-0277

Інститут проблем міцності ім. Г.С. Писаренка НАН України, Київ

E-mail: chirkale82@gmail.com

\title{
Метод пружних розв'язків у задачах радіаційної повзучості, в яких враховуються вплив напружень і накопиченої незворотної деформації на радіаційне розпухання матеріалу
}

Представлено академіком НАН України В.В. Харченком

Розглядається метод пружних розв'язків для розв'язання нелінійних крайових радіаційної повзучості, які дають змогу описувати неізотермічні процеси непружного деформування з урахуванням радіаційного розпухання і радіаційної повзучості опроміненого матеріалу. Для моделювання процесів радіаційного розпухання і радіаційної повзучості застосовуються сучасні підходи, в яких враховується пошкоджуюча доза, температура опромінення, вплив напруженого стану і накопиченої незворотної деформації. Досліджується модифікований метод пружних розв'язків для розв'язання крайових задач радіаційної повзучості. Враховується, що побудова та дослідження властивостей ітераційного методу в задачах радіаційної повзучості ускладнюється тією обставиною, що для доведення збіжності та оцінки точності послідовних наближень необхідно враховувати досить жорстке обмеження, зумовлене з несиметричністю оператора, який пов'язує похибки ітераційного процесу для двох послідовних наближень. За таких умов традиційний підхід дослідження збіжності ітераційного процесу з урахуванням властивостей самоспряжених операторів виявляється неприйнятним. Окрім того, стандартна процедура симетризацї рівняння для послідовних наближень призводить до надмірно консервативних оцінок збіжності ітераційного методy, і тому оптимізація його швидкості збіжності має досить наближений характер. Цю задачу розв'язано завдяки використанню спещіальної норми для аналізу збіжності послідовних наближень, що дозволило побудувати модифікований ітераційний процес та довести його локальну збіжність для загального випадку рівнянь радіаційної повзучості. Докладно вивчено властивості модифікованого процесу $і$ на иій основі одержано апріорні оцінки асимптотичної швидкості збіжності послідовних наближень та сформульовано підходи щодо оптимізації методу пружни розв'язків стосовно задач радіаційної повзучості.

Ключові слова: непружне деформування, радіачійне розпухання, радіачійна повзучість, метод пружних розв'язків, ітерачійний прочес, збіжність і точність послідовних наближень.

Обгрунтування міцності та працездатності елементів конструкцій, що зазнають впливу інтенсивного нейтронного опромінення зумовлено застосуванням у розрахунках напружено-деформованого стану адекватних математичних моделей деформування і методів

Ци т у в анн я: Чирков О.Ю. Метод пружних розв’язків у задачах радіаційної повзучості, в яких враховуються вплив напружень і накопиченої незворотної деформації на радіаційне розпухання матеріалу. Допов. Наи. акад. наук Укр. 2021. № 6. С. 32-44. https://doi.org/10.15407/dopovidi2021.06.032 
розв’язання нелінійних крайових задач механіки, що враховують процеси радіаційного розпухання і радіаційної повзучості металу за умов тривалого опромінення, високих температур та пошкоджуючої дози.

У [1-3] сформульовано визначальні рівняння поведінки опроміненого матеріалу, які дають змогу описувати неізотермічні процеси непружного деформування з урахуванням радіаційного розпухання і радіаційної повзучості, а також досліджено умови, що забезпечують математичну коректність визначальних рівнянь. Використано сучасні підходи до моделювання процесів радіаційного розпухання і радіаційної повзучості, в яких враховується вплив об’ємного напруженого стану та накопиченої незворотної деформації на стиснене розпухання і радіаційну повзучість опроміненого матеріалу [4, 5].

Нижче розглядається узагальнена крайова задача радіаційної повзучості, яку сформульовано у квазістатичній постановці у вигляді нелінійного операторного рівняння для поточного етапу навантаження. На основі одержаних результатів щодо умов коректності визначальних рівнянь радіаційної повзучості встановлено існування, єдиність і безперервна залежність узагальненого розв’язку крайової задачі від зовнішніх впливів.

Відмітимо, що публікації, присвячені методам розв’язання нелінійних крайових задач радіаційної повзучості, обмежуються застосуванням традиційних ітераційних алгоритмів, одним з яких є метод пружних розв’язків. Разом із тим аналіз та оптимізація збіжності процесів пружних розв’язків для розглянутих моделей радіаційного розпухання і радіаційної повзучості не достатньо висвітлені в сучасних публікаціях за даною тематикою. На підставі вищевикладеного можна зробити висновок щодо актуальності розроблення більш досконалого підходу до побудови та аналізу збіжності методу пружних розв’язків для задач радіаційної повзучості, в яких використовується модель радіаційного розпухання з урахуванням напруженого стану і накопиченої незворотної деформації. Розгляду цих питань і присвячено цю статтю. В ній викладено деякі аспекти, пов’язані з модифікацією та оптимізацією послідовних наближень, а також запропоновано підхід для аналізу та доведення збіжності ітераційного процесу пружних розв’язків стосовно задач радіаційної повзучості.

Зазначимо, що ключову роль в аналізі ітераційних процесів становить вибір норми, для якої вдається довести збіжність послідовних наближень за деяких умов, що є загалом нетривіальною математичною задачею. Вона ускладнюється ще й тим, що для рівнянь радіаційної повзучості аналіз збіжності ітераційних процесів обмежений досить жорсткою умовою, яка викликана несиметричністю оператора, що пов’язує похибки ітераційного процесу для двох послідовних наближень. За таких умов традиційні підходи доведення збіжності процесів пружних розв’язків з урахуванням властивостей самоспряжених операторів неприйнятні. Окрім того, стандартна процедура симетризації рівняння для послідовних наближень призводить до надмірно консервативних оцінок збіжності ітераційного процесу, і тому оптимізація його швидкості збіжності має досить наближений характер. Цю задачу розв’язано завдяки застосуванню спеціальної норми до аналізу збіжності послідовних наближень, що дозволило побудувати модифікований ітераційний процес та довести локальну збіжність методу пружних розв’язків для загального випадку рівнянь радіаційної повзучості. 3 використанням одержаних апріорних оцінок сформульовано підходи для оптимізації асимптотичної швидкості збіжності модифікованого методу пружних розв'язків. 
Формулювання узагальненої крайової задачі радіаційної повзучості. Вважаємо, що тіло займає область $\Omega$ у дво- або тривимірному евклідовому просторі та має регулярну границю. На частині границі задані переміщення, що виключають зміщення $\Omega$ як жорсткого тіла, а на решті частини - поверхневі навантаження щільністю $\boldsymbol{f}(\boldsymbol{x}, t)$. Окрім того, тіло знаходиться під впливом пошкоджуючої дози $Z(\boldsymbol{x}, t)$ і температури опромінення $T(\boldsymbol{x}, t)$.

Переміщення тіла описуються компонентами вектор-функції $\boldsymbol{u}(\boldsymbol{x}, t)$, а деформації $\varepsilon(\boldsymbol{x}, t)$ і напруження $\boldsymbol{\sigma}(\boldsymbol{x}, t)$ - симетричними тензор-функціями другого рангу. Початкові деформації $\boldsymbol{\xi}(\boldsymbol{x}, t)$ включають кульові компоненти температурних деформацій і вільного розпухання, а також девіаторні компоненти незворотних деформацій, накопичених на початок етапу навантаження. Окрім того, до початкових деформацій додаються об'ємні структурні деформації э $(\boldsymbol{x}, t)$, що враховують вплив інтенсивності напружень на радіаційне розпухання матеріалу. Вище позначено: $\boldsymbol{x}-$ координати точки тіла, $t-$ час або інший параметр, що характеризує процес навантаження.

Вважаємо, що переміщення є елементами простору $U$, що складається з вектор-функцій, які інтегруються з квадратом на $\Omega$ разом зі своїми першими похідними включно і дорівнюють нулю на частині границі тіла. Напруження і деформації будемо розглядати як елементи простору $L$ тензор-функцій, інтегрованих з квадратом на $\Omega$ та нормою, асоційованою зі скалярним добутком

$$
(\sigma, \varepsilon)_{L}=\int_{\Omega}(\sigma, \varepsilon) d \Omega
$$

де $(\sigma, \varepsilon)=\sigma_{\mathrm{ij}} \varepsilon_{\mathrm{ij}}-$ згортка тензорів напружень і деформацій.

Далі будемо розглядати довільні тензори напружень і деформацій в точці тіла, як елементи евклідового простору $\mathbb{E}$, в якому скалярний добуток визначається згорткою відповідних тензорів (·, ·). Нормою, асоційованою з цим скалярним добутком, є модуль тензора $\|\cdot\|$.

Узагальнену крайову задачу радіаційної повзучості у квазістатичній постановці для будьякого фіксованого значення параметра $t>0$ сформулюємо наступним чином. Знайти трійку $(\boldsymbol{u}(t), \varepsilon(t), \sigma(t)) \in U \times L \times L$ таку, що

$$
\begin{aligned}
& (\varepsilon(t), \delta \sigma)_{L}=(\mathcal{B} \boldsymbol{u}(t), \delta \sigma)_{L}, \quad \forall \delta \sigma \in L ; \\
& (\sigma(t), \delta \varepsilon)_{L}=(\mathcal{P}(\varepsilon(t), \xi(t)), \delta \varepsilon)_{L}, \quad \forall \delta \varepsilon \in L ; \\
& (\sigma(t), \mathcal{B} \delta \boldsymbol{u})_{L}=\langle\boldsymbol{f}(t), \delta \boldsymbol{u}\rangle, \quad \forall \delta \boldsymbol{u} \in U,
\end{aligned}
$$

де $\delta \boldsymbol{u}, \delta \varepsilon, \delta \sigma$ - довільні неперервні функції, які можна інтерпретувати як варіації напружень, деформацій і переміщень; $\mathcal{B}$ - лінійний диференційний оператор обчислення малих деформацій $\varepsilon(t)$ за заданими переміщеннями $\boldsymbol{u}(t) ; \mathcal{P}-$ нелінійний оператор, що встановлює зв'язок напружень з деформаціями; $\langle\boldsymbol{f}(t), \delta \boldsymbol{u}\rangle-$ лінійна форма, яка тотожна роботі поверхневих навантажень на можливих переміщеннях $\delta \boldsymbol{u} \in U$.

Нелінійний оператор $\mathcal{P}$ діє в просторі $L$ і для ізотропного матеріалу визначається за співвідношенням

$$
\begin{aligned}
& \mathcal{P}(\varepsilon(t), \xi(t))=k_{Q}^{R}(T(t))\left(\varepsilon_{S}(t)-\ni\left(\varepsilon_{D}(t)\right)-\xi_{S}(t)\right)+ \\
& +2 G_{s}^{R}(\bar{\varepsilon}(\varepsilon(t), \xi(t)), \mathrm{Z}(t), T(t))\left(\varepsilon_{D}(t)-\xi_{D}(t)\right),
\end{aligned}
$$


де $k_{Q}^{R}$ - модуль всебічного об’ємного розширення з урахуванням поправки на радіаційне розпухання і накопичену незворотну деформацію матеріалу на початок етапу навантаження; $G_{s}^{R}-$ січний модуль зсуву, що враховує вплив радіаційної повзучості за етап навантаження; $\bar{\varepsilon}^{a}-$ інтенсивність девіатора активних деформацій, які виникають у точці тіла додатково до початкових незворотних деформацій наприкінці етапу навантаження. Кульову і девіаторну складові довільного тензора деформацій відзначено нижніми символами $S$ і $D$ відповідно. Ці та інші використовувані нижче позначення детально наведені в [1-3].

Рівняння (2) дають змогу сформулювати узагальнену крайову задачу в переміщеннях:

$$
(\mathcal{P}(\varepsilon(t), \xi(t)), \mathcal{B} \delta \boldsymbol{u})_{L}=\langle\boldsymbol{f}(t), \delta \boldsymbol{u}\rangle, \quad \forall \delta \boldsymbol{u} \in U
$$

Отже, крайову задачу (4) можна записати у вигляді нелінійного операторного рівняння

$$
\mathcal{A}(\boldsymbol{u}(t), \xi(t))=\boldsymbol{f}(t) ; \quad \boldsymbol{u}(t) \in U, \quad \boldsymbol{f}(t) \in U^{*}
$$

де $\mathcal{A}: U \rightarrow U^{*}-$ нелінійний оператор, який визначається відображенням

$$
\mathcal{A}: \boldsymbol{u} \in U \rightarrow(\mathcal{P}(\mathcal{B} \boldsymbol{u}(t), \xi(t)), \mathcal{B} \boldsymbol{w})_{L}, \quad \forall \boldsymbol{w} \in U
$$

На основі результатів щодо коректності визначальних рівнянь, одержаних в [2, 3], приходимо до висновку: нелінійний оператор $\mathcal{A}$ має властивості сильної монотонності та ліпшиць-неперервності [6]. Звідси випливає однозначний розв'язок операторного рівняння (5), а також його безперервна залежність від заданих навантажень $f(t) \in U^{*}$ та початкових деформацій $\xi(t) \in L$.

Метод пружних розв'язків. В узагальненому методі пружних розв'язків послідовність лінійних наближень $\left\{\boldsymbol{u}^{k}\right\}_{k=1}^{\infty}$ будується на кожному етапі навантаження у вигляді такої ітераційної процедури

$$
\boldsymbol{u}^{k+1}=\boldsymbol{u}^{k}-\alpha \mathcal{K}_{0}^{-1}\left(\mathcal{A}\left(\boldsymbol{u}^{k}, \xi\right)-\boldsymbol{f}\right)
$$

де $\alpha>0$ - числовий параметр, що вводиться для управління збіжністю процесу, який може змінюватися від ітерації до ітерації; $\mathcal{K}_{0}-$ лінійний коерцитивний оператор для лінійнопружної задачі, який здійснює відображення з $U$ в $U^{*}$ :

$$
\mathcal{K}_{0}: \boldsymbol{u} \in U \rightarrow\left(\mathcal{D}_{0} \mathcal{B} \boldsymbol{u}, \mathcal{B} \boldsymbol{w}\right)_{L}, \quad \forall \boldsymbol{w} \in U
$$

$\mathcal{D}_{0}$ - лінійний самоспряжений додатно визначений оператор початкових модулів пружності, які визначаються з урахуванням радіаційного розпухання і радіаційної повзучості матеріалу:

$$
\mathcal{D}_{0} \mu=k_{Q}^{R} \mu_{S}+2 G_{0}^{R} \mu_{D}, \quad \forall \mu \in L .
$$

де $G_{0}^{R}$ - початковий модуль зсуву, що враховує вплив радіаційної повзучості за етап навантаження на пружній ділянці діаграми деформування матеріалу.

У разі зіставлення рівнянь (5), (7) одержуємо співвідношення для похибок ітераційного процесу 


$$
\boldsymbol{u}^{k+1}-\boldsymbol{u}=\boldsymbol{u}^{k}-\boldsymbol{u}-\alpha \mathcal{K}_{0}^{-1}\left(\mathcal{A}\left(\boldsymbol{u}^{k}, \xi\right)-\mathcal{A}(\boldsymbol{u}, \xi)\right)
$$

3 якого випливає

$$
\left(\mathcal{D}_{0} \mathcal{B}\left(\boldsymbol{u}^{k+1}-\boldsymbol{u}\right), \mathcal{B} \boldsymbol{w}\right)_{L}=\left(\mathcal{D}_{0} \mathcal{B}\left(\boldsymbol{u}^{k}-\boldsymbol{u}\right), \mathcal{B} \boldsymbol{w}\right)_{L}-\alpha\left(\mathcal{P}\left(\mathcal{B} \boldsymbol{u}^{k}\right)-\mathcal{P}(\mathcal{B} \boldsymbol{u}), \mathcal{B} \boldsymbol{w}\right)_{L}, \quad \forall \boldsymbol{w} \in U
$$

Оскільки $\mathcal{D}_{0}$ - самоспряжений додатно визначений оператор, його можна використовувати для побудови в просторі $L$ скалярного добутку $(\cdot, \cdot)_{\mathcal{D}_{0}}$ і енергетичної норми $\|\cdot\|_{\mathcal{D}_{0}}$, яка еквівалентна вихідній нормі цього простору.

Якщо в рівнянні (11) покласти $\boldsymbol{w}=\boldsymbol{u}^{k}-\boldsymbol{u} \in U$ то з використанням формули скінченних приростів і нерівності Коши-Буняковського-Шварца [6] маємо

$$
\left\|\varepsilon^{k+1}-\varepsilon\right\|_{\mathcal{D}_{0}} \leqslant \sup _{\boldsymbol{e} \in L}\left\|\mathcal{T}_{\alpha}^{\prime}(\boldsymbol{e})\right\|_{\mathcal{D}_{0}}\left\|\varepsilon^{k}-\varepsilon\right\|_{\mathcal{D}_{0}}
$$

де $\mathcal{T}_{\alpha}^{\prime}(\boldsymbol{e})-$ значення похідної (за Фреше) оператора

$$
\mathcal{T}_{\alpha}: \boldsymbol{e} \in L \rightarrow \mathcal{T}_{\alpha}(\boldsymbol{e})=\boldsymbol{e}-\alpha \mathcal{D}_{0}^{-1} \mathcal{P}(\boldsymbol{e})
$$

у довільній точці $\boldsymbol{e} \in L$. Отже, оператор $\mathcal{T}_{\alpha}^{\prime}(\boldsymbol{e})$ визначається виразом:

$$
\mathcal{T}_{\alpha}^{\prime}(\boldsymbol{e}) \mu=\mu-\alpha \mathcal{D}_{0}^{-1} \mathcal{P}^{\prime}(\boldsymbol{e}) \mu, \quad \forall \mu \in L
$$

Відмітимо, що доведення та оцінка збіжності класичного методу пружних розв’язків для задач деформаційної теорії пластичності одержані з урахуванням умови, що $\mathcal{P}^{\prime}-$ самоспряжений оператор у просторі $L$ для заданого елемента $\boldsymbol{e} \in L$. Ця властивість оператора $\mathcal{P}^{\prime}$ істотно спрощує аналіз збіжності ітераційного процесу, тому що призводить до оцінки $\mathcal{D}_{0}$-норми самоспряженого оператора $\mathcal{T}_{\alpha}^{\prime}$.

Для визначальних рівнянь радіаційної повзучості зазначена властивість оператора $\mathcal{P}^{\prime}$ не виконується [3], і тому ключову роль в аналізі збіжності процесу пружних розв'язків становить вибір норми оператора $\mathcal{T}_{\alpha}^{\prime}$, яку необхідно оцінити. Заміна норми вихідного простору $L$ на еквівалентну не змінює значення оператора $\mathcal{T}_{\alpha}^{\prime}$, хоча величина самої норми змінюється. Нижню межу з усіх можливих оцінок норм оператора $\mathcal{T}_{\alpha}^{\prime}$ дає множник збіжності за коренями $r(\boldsymbol{u})$.

Використання $\mathcal{D}_{0}$-норми для дослідження збіжності послідовних наближень дозволяє встановити умови, що забезпечують збіжність методу пружних розв'язків незалежно від вибору початкового наближення. Проте одержані апріорні оцінки виявляються надмірно консервативними, а їх застосування до оптимізації швидкості збіжності ітераційного процесу має досить наближений характер.

Зазначимо, що для доведення локальної збіжності методу пружних розв’язків необхідно встановити, що існує норма $\|\cdot\|_{H}$, еквівалентна вихідній нормі простору $L$, для якої виконується умова $r(\boldsymbol{u}) \leqslant q<1$. Тоді для будь-якого, але досить близького до $\boldsymbol{u}$ початкового наближення $\boldsymbol{u}^{0} \in U$, послідовність $\left\{\boldsymbol{u}^{k}\right\}_{k=1}^{\infty}$, яка побудована за допомогою ітераційного процесу (7), збігається до розв’язку рівняння (5). Для загального випадку рівнянь радіаційної повзучості оцінку локальної збіжності методу пружних розв’язків можна отримати 3 використанням оператора $H$ у вигляді $H=\mathcal{D}_{0}^{2}$. Дійсно, оскільки $H-$ лінійний самоспря- 
жений додатно визначений оператор, його можна використовувати для побудови у просторі $L$ скалярного добутку $(\cdot, \cdot)_{H}$ та норми $\|\cdot\|_{H}$. Тоді асимптотичну швидкість збіжності процесу пружних розв’язків в околі кореня $\boldsymbol{u}$ можна оцінити з застосуванням множника збіжності за коренями $r(\boldsymbol{u}) \leqslant q<1$, причому збіжність послідовних наближень характеризується нерівностями [6]:

$$
\mid \varepsilon^{k}-\varepsilon\left\|_{H} \leqslant q\right\| \varepsilon^{k-1}-\varepsilon\left\|_{H} \leqslant \cdots \leqslant q^{k}\right\| \varepsilon^{0}-\varepsilon \|_{H} .
$$

За таким підходом вдається покращити оцінки асимптотичної швидкості збіжності методу пружних розв'язків, проте одержані апріорні оцінки як і раніше залишаються достатньо консервативними, тому їх застосування до оптимізації ітераційного процесу не призводить до істотного покращення збіжності.

Модифікований ітераційний процес. Розглянемо модифікований метод пружних розв’язків, в якому послідовність наближень будується у вигляді (7), однак за умови, що параметр $\alpha$ дорівнює одиниці, а лінійний оператор $\mathcal{D}_{0}$ залежить від параметра $\lambda>0$ i визначається наступним чином:

$$
\mathcal{D}_{0}(\lambda) \mu=k_{Q}^{R} \mu_{S}+2 \lambda G_{0}^{R} \mu_{D}, \quad \forall \mu \in L .
$$

У рівнянні (16) використовується модуль всебічного об’ємного розширення $k_{Q}^{R}$ з урахуванням поправки на стиснене розпухання і накопичену незворотну деформацію $Q$ матеріалу на початок поточного етапу навантаження:

$$
k_{Q}^{R}=\frac{k_{0}}{1+\frac{1}{3} k_{0} R_{0} C_{R} \omega e^{-\gamma Q}},
$$

де $k_{0}$ - початковий модуль всебічного об’ємного розширення, який залежить від дози і температури опромінення матеріалу; $R_{0}-$ вільне розпухання без напружень; $C_{R}$ i $\gamma-$ константи матеріалу, які визначаються за експериментальними даними; $\omega \in[0,1]-$ ваговий множник, що визначає ступінь впливу середнього нормального та інтенсивного напружень на стиснене розпухання матеріалу $R$. Коефіцієнт $\omega$ одержаний за допомогою обробки експериментальних даних із визначення розпухання методом найменших квадратів, $\omega=\omega_{0}=0,85[4,5]$.

Модифікація процесу пружних розв’язків з використанням перетворення (16) сприяє покращенню швидкості збіжності послідовних наближень, тому що для оцінки $\mathcal{D}_{0}(\lambda)$-норми оператора $\mathcal{T}_{\alpha}^{\prime}$ достатньо розглядати не всю множину можливих деформацій, а більш вузьку підмножину, яка складається з девіаторних компонент тензорів деформацій.

Зазначимо, що подібну модифікацію методу пружних розв'язків, в якій замість реального початкового модуля зсуву $G_{0}$ використовується видозмінений модуль $\tilde{G}_{0}$, застосовують до розв’язання задач деформаційної теорії пластичності. Для певного вибору співвідношення $\tilde{G}_{0} / G_{0}$ можна одержати більш оптимальну оцінку, тобто покращити швидкість збіжності ітераційного процесу порівняно з класичним методом пружних розв’язків.

Для загального випадку рівнянь радіаційної повзучості доведення збіжності модифікованого методу пружних розв’язків враховує важливу властивість оператора $\mathcal{P}^{\prime}$, яке полягає у наступному. Оператор $\mathcal{P}^{\prime}$ за визначенням включає самоспряжений оператор, що 
враховує вплив середнього нормального напруження на розпухання. 3 використанням цієї властивості оператора $\mathcal{P}^{\prime}$ можна одержати більш оптимальну оцінку асимптотичної швидкості збіжності ітераційного процесу порівняно із загальним підходом.

Теорема. Якщо $H(\lambda)$-норма визначається співвідношенням $H(\lambda)=\mathcal{D}_{0}{ }^{2}(\lambda)$, оператор $\mathcal{T}_{\lambda}^{\prime}$ задовольняє умові збіжності $\left\|\mathcal{T}_{\lambda}^{\prime}(\boldsymbol{e})\right\|_{H} \leqslant q(\lambda)$, де множник $q(\lambda)$ обчислюється за формулою

$$
q^{2}(\lambda)=\max \left(1-2 \lambda^{-1} \theta_{0}+\lambda^{-2} \theta_{0}^{2}\left(1+\phi_{0}^{2}\right), 1-2 \lambda^{-1}+\lambda^{-2}\left(1+\phi_{0}^{2}\right)\right),
$$

де $\theta_{0}$ - параметр, що задається відношенням дотичного $G_{\tau}^{R}$ до початкового $G_{0}^{R}$ модуля зсуву опроміненого матеріалу,

$$
\theta=\frac{G_{\tau}^{R}}{G_{0}^{R}} \leqslant 1, \quad \theta_{0}=\text { ess. } \inf _{\boldsymbol{x} \in \Omega} \theta(\boldsymbol{x}) ;
$$

$\phi_{0}-$ числовий параметр, який оцінюється так: $\phi_{0} \approx 0,375$.

Доведення. Оскільки $H(\lambda)$ - лінійний самоспряжений додатно визначений оператор, його можна використати для побудови в просторі $L$ скалярного добутку і норми, яка еквівалентна вихідній нормі цього простору:

$$
\begin{aligned}
& (\mu, \eta)_{H}=(H(\lambda) \mu, \eta)_{L}=\left(\mathcal{D}_{0}(\lambda) \mu, \mathcal{D}_{0}(\lambda) \eta\right)_{L}, \quad \forall \mu, \eta \in L ; \\
& \|\mu\|_{H}=(\mu, \mu)_{H}^{1 / 2}, \quad \forall \mu \in L .
\end{aligned}
$$

Тоді $H(\lambda)$-норма оператора $\mathcal{T}_{\lambda}^{\prime}$ визначається рівністю

$$
\left\|\mathcal{T}_{\lambda}^{\prime}(\boldsymbol{e})\right\|_{H}=\sup _{\mu \in L} \frac{\left\|\mathcal{T}_{\lambda}^{\prime}(\boldsymbol{e}) \mu\right\|_{H}}{\|\mu\|_{H}} .
$$

Якщо елемент $\mu \in L$ у правій частині (21) записати у вигляді $\mu=\mathcal{D}_{0}^{-1}(\lambda) \eta$, приходимо до співвідношення

$$
\left\|\tilde{\mathcal{T}}_{\lambda}^{\prime}(\boldsymbol{e})\right\|_{H}=\sup _{\eta \in L} \frac{\left\|\tilde{\mathcal{T}}_{\lambda}^{\prime}(\boldsymbol{e}) \eta\right\|_{L}}{\|\eta\|_{L}}
$$

де оператор $\tilde{\mathcal{T}}_{\lambda}^{\prime}$ визначається за формулою:

$$
\tilde{\mathcal{T}}_{\lambda}^{\prime}(\boldsymbol{e}) \eta=\mathcal{D}_{0}(\lambda) \mathcal{T}_{\lambda}^{\prime}(\boldsymbol{e}) \mathcal{D}_{0}^{-1}(\lambda) \eta, \quad \forall \eta \in L
$$

Згідно з (14), (23) для будь-якого $\eta \in L$ маємо

$$
\tilde{\mathcal{T}}_{\lambda}^{\prime}(\boldsymbol{e}) \eta=\eta-\alpha \mathcal{P}^{\prime}(\boldsymbol{e}) \mathcal{D}_{0}^{-1}(\lambda) \eta,
$$

де оператор $\mathcal{P}^{\prime}$ визначається наступним чином [3]:

$$
\begin{aligned}
& \mathcal{P}^{\prime}(\boldsymbol{e}) \mu=k_{Q}^{R} \mu_{S}+2 G_{s}^{R} \mu_{D}+2\left(G_{\tau}^{R}-G_{s}^{R}\right) \frac{\left(\boldsymbol{e}_{D}, \mu_{D}\right)}{\left\|\boldsymbol{e}_{D}\right\|^{2}} \boldsymbol{e}_{D}- \\
& -\sqrt{\frac{2}{3}} k_{Q}^{R} G_{\tau}^{R} R_{0} C_{R}(1-\omega) e^{-\gamma Q} \frac{\left(\boldsymbol{e}_{D}, \mu_{D}\right)}{\left\|\boldsymbol{e}_{D}\right\|} \boldsymbol{s}_{1}, \quad \forall \mu \in L,
\end{aligned}
$$


де $\boldsymbol{s}_{1}-$ одиничний кульовий тензор, його модуль дорівнює $\sqrt{3}$.

Оскільки оператор $\mathcal{D}_{0}^{-1}(\lambda)$ можна подати у вигляді

$$
\mathcal{D}_{0}^{-1}(\lambda) \eta=\frac{1}{k_{Q}^{R}} \eta_{S}+\frac{1}{2 \lambda G_{0}^{R}} \eta_{D}, \quad \forall \eta \in L,
$$

на підставі співвідношень (24)-(26) одержимо

$$
\begin{aligned}
& \tilde{\mathcal{T}}_{\lambda}^{\prime}(\boldsymbol{e}) \eta=\left(1-\frac{\rho}{\lambda}\right) \eta_{D}-\frac{1}{\lambda}(\theta-\rho) \frac{\left(\boldsymbol{e}_{D}, \eta_{D}\right)}{\left\|\boldsymbol{e}_{D}\right\|^{2}} \boldsymbol{e}_{D}+ \\
& +\frac{1}{\sqrt{6}} \frac{\theta}{\lambda} k_{Q}^{R} R_{0} C_{R}(1-\omega) e^{-\gamma Q} \frac{\left(\boldsymbol{e}_{D}, \eta_{D}\right)}{\left\|\boldsymbol{e}_{D}\right\|} \boldsymbol{s}_{1}, \quad \forall \eta \in L,
\end{aligned}
$$

де $\rho$ - параметр, який визначається в точці тіла, як відношення січного до початкового модуля зсуву опроміненого матеріалу:

$$
\rho=\frac{G_{s}^{R}}{G_{0}^{R}} \leqslant 1, \quad \theta-\rho \leqslant 0 .
$$

3 формули (27) випливає, що областю визначення оператора $\tilde{\mathcal{T}}_{\lambda}^{\prime} \in$ не вся множина можливих деформацій $\mathbb{E}$, а тільки ії більш вузька підмножина $\mathbb{E}_{D}$, яка складається з девіаторних компонент тензорів деформацій.

Отже, норма оператор $\tilde{\mathcal{T}}_{\lambda}^{\prime}$ визначається за формулою:

$$
\left\|\mathcal{T}_{\lambda}^{\prime}(\boldsymbol{e})\right\|_{H}=\sup _{\eta_{D} \in L} \frac{\left\|\tilde{\mathcal{T}}_{\lambda}^{\prime}(\boldsymbol{e}) \eta_{D}\right\|_{L}}{\left\|\eta_{D}\right\|_{L}}
$$

Згідно з (27) оператор $\tilde{\mathcal{T}}_{\lambda}^{\prime}(\boldsymbol{e})$ можемо записати у вигляді суми двох операторів

$$
\tilde{\mathcal{T}}_{\lambda}^{\prime}(\boldsymbol{e}) \eta_{D}=\mathcal{M}_{\lambda}(\boldsymbol{e}) \eta_{D}+\mathcal{N}_{\lambda}(\boldsymbol{e}) \eta_{D}, \quad \forall \eta_{D} \in \mathbb{E}_{D}
$$

де $\mathcal{M}_{\lambda}-$ самоспряжений оператор,

$$
\mathcal{M}_{\lambda}(\boldsymbol{e}) \eta_{D}=\left(1-\frac{\rho}{\lambda}\right) \eta_{D}-\frac{1}{\lambda}(\theta-\rho) \frac{\left(\boldsymbol{e}_{D}, \eta_{D}\right)}{\left\|\boldsymbol{e}_{D}\right\|^{2}} \boldsymbol{e}_{D} ;
$$

$\mathcal{N}_{\lambda}$ - несамоспряжена частина оператора $\tilde{\mathcal{T}}_{\lambda}^{\prime}$,

$$
\mathcal{N}_{\lambda}(\boldsymbol{e}) \eta_{D}=\frac{1}{\sqrt{6}} \frac{\theta}{\lambda} k_{Q}^{R} R_{0} C_{R}(1-\omega) e^{-\gamma Q} \frac{\left(\boldsymbol{e}_{D}, \eta_{D}\right)}{\left\|\boldsymbol{e}_{D}\right\|} \boldsymbol{s}_{1}
$$

На підставі співвідношень (30)-(31) приходимо до рівності

$$
\left\|\tilde{\mathcal{T}}_{\lambda}^{\prime}(\boldsymbol{e}) \eta_{D}\right\|^{2}=\left\|\mathcal{M}_{\lambda}(\boldsymbol{e}) \eta_{D}\right\|^{2}+\left\|\mathcal{N}_{\lambda}(\boldsymbol{e}) \eta_{D}\right\|^{2},
$$


де враховано, що $\left(\boldsymbol{s}_{1}, \eta_{D}\right)=0$ і тому виконується умова

$\left(\mathcal{M}_{\lambda}(\boldsymbol{e}) \eta_{D}, \mathcal{N}_{\lambda}(\boldsymbol{e}) \eta_{D}\right)=0, \quad \forall \eta_{D} \in \mathbb{E}_{D}$

Оцінимо зверху перший доданок у формулі (33) на підставі нерівності

$$
\left\|\mathcal{M}_{\lambda}(\boldsymbol{e}) \eta_{D}\right\| \leqslant\left\|\mathcal{M}_{\lambda}(\boldsymbol{e})\right\|\left\|\eta_{D}\right\|, \quad \forall \eta_{D} \in \mathbb{E}_{D}
$$

3 використанням (31) для будь-яких $\eta_{D}, \chi_{D} \in \mathbb{E}_{D}$ маємо

$$
\left(\mathcal{M}_{\lambda}(\boldsymbol{e}) \eta_{D}, \chi_{D}\right)=\left(1-\frac{\rho}{\lambda}\right)\left(\eta_{D}, \chi_{D}\right)-\frac{1}{\lambda}(\theta-\rho) \frac{\left(\boldsymbol{e}_{D}, \eta_{D}\right)\left(\boldsymbol{e}_{D}, \chi_{D}\right)}{\left\|\boldsymbol{e}_{D}\right\|^{2}} .
$$

Останнє співвідношення симетричне відносно елементів $\eta_{D} \mathrm{i} \chi_{D}$, тобто переконуємося, що $\mathcal{M}_{\lambda}-$ самоспряжений оператор у $\mathbb{E}_{D}$ i тому його норма визначається за формулою

$$
\left\|\mathcal{M}_{\lambda}(\boldsymbol{e})\right\|=\sup _{\eta_{D} \in \mathbb{E}_{D}} \frac{\left|\left(\mathcal{M}_{\lambda}(\boldsymbol{e}) \eta_{D}, \eta_{D}\right)\right|}{\left\|\eta_{D}\right\|^{2}} .
$$

Для оцінки норми оператора $\mathcal{M}_{\lambda}$ запишемо рівність

$$
\left(\mathcal{M}_{\lambda}(\boldsymbol{e}) \eta_{D}, \eta_{D}\right)=\left(1-\frac{\rho}{\lambda}\right)\left\|\eta_{D}\right\|^{2}-\frac{1}{\lambda}(\theta-\rho) \frac{\left(\boldsymbol{e}_{D}, \eta_{D}\right)^{2}}{\left\|\boldsymbol{e}_{D}\right\|^{2}},
$$

звідки з урахуванням умови $\theta-\rho \leqslant 0$, а також нерівності Коші-Буняковського-Шварца [6] одержуємо

$$
\left\|\mathcal{M}_{\lambda}(\boldsymbol{e})\right\| \leqslant \max \left(1-\frac{\theta}{\lambda}, \frac{\rho}{\lambda}-1\right)
$$

Оцінимо зверху другий доданок у рівності (33), який визначається за формулою (32). 3 використанням нерівності Коші-Буняковського-Шварца для будь-якого $\eta_{D} \in \mathbb{E}_{D}$ маємо

$$
\left\|\mathcal{N}_{\lambda}(\boldsymbol{e}) \eta_{D}\right\| \leqslant \frac{1}{\sqrt{2}} \frac{\theta}{\lambda} k_{Q}^{R} R_{0} C_{R}(1-\omega) e^{-\gamma Q}\left\|\eta_{D}\right\|
$$

Врахуємо в останній нерівності оцінку, що випливає з рівності (17):

$$
k_{Q}^{R} R_{0} C_{R} e^{-\gamma Q}=\frac{3}{\omega}\left(1-\frac{k_{Q}^{R}}{k_{0}}\right) \leqslant \frac{3}{\omega} .
$$

Тоді приходимо до наступної оцінки:

$$
\left\|\mathcal{N}_{\lambda}(\boldsymbol{e}) \eta_{D}\right\| \leqslant \frac{\theta}{\lambda} \phi(\omega)\left\|\eta_{D}\right\|, \quad \forall \eta_{D} \in \mathbb{E}_{D}
$$

де числовий параметр $\phi(\omega)$ визначається за формулою:

$$
\phi(\omega)=\frac{3}{\sqrt{2}} \frac{1-\omega}{\omega} \geqslant 0 .
$$


У разі вибору $\omega=\omega_{0}=0,85$ знаходимо $\phi_{0}=\phi\left(\omega_{0}\right)<0,375$.

Фактично за теоремою Піфагора, а саме відповідно до рівності (33) та оцінкам (35), (39), (42), приходимо до нерівності

$$
\left\|\tilde{\mathcal{T}}_{\lambda}^{\prime}(\boldsymbol{e}) \eta_{D}\right\| \leqslant q(\lambda)\left\|\eta_{D}\right\|, \quad \forall \eta_{D} \in \mathbb{E}_{D},
$$

де множник $q(\lambda)$ визначається формулою (18).

Таким чином, виходячи із співвідношень (29) та (44), приходимо до оцінки $H(\lambda)$-норми оператора $\mathcal{T}_{\lambda}^{\prime}$ :

$$
\sup _{\boldsymbol{e} \in L}\left\|\mathcal{T}_{\lambda}^{\prime}(\boldsymbol{e})\right\|_{H} \leqslant q(\lambda)
$$

Теорема доведена.

Наслідок 1. 3 виразу (18) випливає, що умова $q(\lambda)<1$ буде виконуватися тільки в тому разі, якщо параметр $\lambda$ задовольняє нерівності

$$
\lambda>\frac{1}{2}\left(1+\phi_{0}^{2}\right) \approx 0,57
$$

За таких умов розв'язок $\boldsymbol{u}$ рівняння (5) є точкою тяжіння модифікованого процесу пружних розв'язків, причому асимптотичну швидкість збіжності послідовних наближень в околі кореня $\boldsymbol{u}$ можна оцінити за допомогою множника збіжності за коренями $r(\boldsymbol{u}) \leqslant q<1$.

Наслідок 2. Оптимальне значення $\lambda_{\text {opt }} \in$ розв'язком рівняння

$$
1-2 \lambda_{\text {opt }}^{-1} \theta_{0}+\lambda_{\text {opt }}^{-2} \theta_{0}^{2}\left(1+\phi_{0}^{2}\right)=1-2 \lambda_{\text {opt }}^{-1}+\lambda_{\text {opt }}^{-2}\left(1+\phi_{0}^{2}\right)
$$

та обчислюється за формулою

$$
\lambda_{\text {opt }}=\frac{1}{2}\left(1+\theta_{0}\right)\left(1+\phi_{0}^{2}\right) .
$$

Підставляючи значення $\lambda_{\text {opt }}$ в рівність (18), одержимо

$$
q^{2}\left(\lambda_{\text {opt }}\right)=1-\frac{4 \theta_{0}}{\left(1+\theta_{0}\right)^{2}\left(1+\phi_{0}^{2}\right)}<1 .
$$

Наслідок 3. Якщо в процесі деформування матеріалу пластичні деформації не виникають або вони настільки малі, що ними можна знехтувати, то в такому разі у формулах (48), (49) можемо покласти $\theta_{0}=1$, звідки знаходимо

$$
\lambda_{o p t}=1+\phi_{0}^{2} \approx 1,14 ; \quad q\left(\lambda_{o p t}\right)=\frac{\phi_{0}}{\sqrt{1+\phi_{0}^{2}}}<0,352 .
$$

Наслідок 4. Оптимальна оцінка асимптотичної швидкості збіжності модифікованого методу пружних розв’язків в околі кореня $\boldsymbol{u}$ має вигляд 


$$
\left\|\varepsilon^{k}-\varepsilon\right\|_{L} \leqslant \frac{d_{02}}{d_{01}} q^{k}\left(\lambda_{o p t}\right)\left\|\varepsilon^{0}-\varepsilon\right\|_{L}
$$

де сталі $d_{01}$ i $d_{02}$ задаються за співвідношеннями:

$$
\begin{array}{ll}
d_{1}=\min \left(2 G_{0}^{R}, k_{Q}^{R}\right)>0 ; & d_{2}=\max \left(2 G_{0}^{R}, k_{Q}^{R}\right)<k_{0} ; \\
d_{01}=\text { ess. } \inf _{\boldsymbol{x} \in \Omega} d_{1}(\boldsymbol{x})>0 ; & d_{02}=\lambda_{\text {opt }} \text { ess. } \sup _{\boldsymbol{x} \in \Omega} d_{2}(\boldsymbol{x}) .
\end{array}
$$

Зауваження 1. Визначення оператора $\mathcal{D}_{0}$ грунтується на використанні початкового модуля зсуву $G_{0}^{R}$ опроміненого матеріалу, що враховує радіаційну повзучість залежно від приростів пошкоджуючої дози та радіаційного розпухання матеріалу за етап навантаження. Однак розпухання є шуканою функцією, і тому на початковому етапі розв'язання задачі вона невідома. Коригувати модуль зсуву в процесі пружних розв’язків залежно від розпухання недоцільно, тому що його уточнення методом змінних параметрів пружності приводить до більш ефективної ітераційної процедури. У такому разі для завдання модуля $G_{0}^{R}$ достатньо використовувати лише приріст пошкоджуючої дози за етап навантаження, що цілком є прийнятним, тому що навіть для такого визначення модуля зсуву ітераційний процес досягає досить високої швидкості збіжності в практичних розрахунках. Отже, для кожного етапу навантаження модуль зсуву визначається лише один раз на початку розв’язання задачі. Можливо також використовувати початковий модуль зсуву неопроміненого матеріалу, який не залежить від пошкоджуючої дози та розпухання, однак це дещо погіршує збіжність послідовних наближень у разі використання протяжних етапів навантаження.

Зауваження 2. Наведені оцінки збіжності методу пружних розв’язків не враховують похибку обчислення початкових деформацій $\xi$, які залежать від історії непружного деформування матеріалу. Початкові деформації $\xi$ на кожному етапі навантаження визначаються за результатами розв’язання крайової задачі на попередньому етапі навантаження і тому включають похибку, яка зумовлена наближеним розв’язком рівняння (5) на кожному етапі навантаження. Фактично на кожному етапі навантаження замість рівняння (5) розв’язується наближене рівняння, і тому одержані оцінки збіжності методу пружних розв’язків встановлюють збіжність послідовних наближень саме до розв’язку наближеного рівняння.

Аналіз збіжності однокрокових ітераційних методів розв'язання нелінійних крайових задач механіки непружного деформування, в яких враховується історія навантаження, а також доведення оцінки збіжності послідовних наближень з урахуванням наближеного визначення незворотних деформацій за результатами розрахунків на попередніх етапах навантаження буде викладено в наступному повідомленні. Ці результати узагальнюють аналіз збіжності методу пружних розв’язків стосовно розв’язання задач радіаційного повзучості.

\section{ЦИТОВАНА ЛІТЕРАТУРА}

1. Чирков О.Ю. Аналіз моделей радіаційного розпухання і радіаційної повзучості, в яких враховується вплив напружень, у задачах механіки непружного деформування. Повідомлення 1. Формулювання визначальних рівнянь. Пробл. міциноті. 2021. № 2. С. 5-17. https://doi.org /10.1007/s11223-021-00276-0 
2. Чирков О.Ю. Аналіз коректності задач механіки непружного деформування, що враховують вплив напруженого стану на процеси радіаційного розпухання і радіаційної повзучості матеріалу. Допов. Нац. акад. наук Укр. 2021. № 2. С. 29-37. https://doi.org /10.15407/dopovidi2021.02.029

3. Чирков О.Ю. Коректність рівнянь радіаційної повзучості, що враховують напруження і накопичену незворотну деформацію в моделі радіаційного розпухання опроміненого матеріалу. Допов. Нац. акад. наук Укр. 2021. № 4. С. 36-45. https://doi.org /10.15407/dopovidi2021.04.036

4. Сорокин А.А., Марголин Б.З., Курсевич И.П. и др. Влияние нейтронного облучения на механические свойства материалов внутрикорпусных устройств реакторов типа ВВЭР. Вопр. материаловедения. 2011. № 2 (66). C. 131-151.

5. Марголин Б.З., Мурашова А.И., Неустроев В.С. Анализ влияния вида напряженного состояния на радиационное распухание и радиационную ползучесть аустенитных сталей. Пробл. прочности. 2012. № 3. C. $5-24$.

6. Ortega J.M., Rheinboldt W.C. Iterative Solution if Nonlinear Equations in Several Variables. New York; London: Academic Press. 1970. 592 p.

Надійшло до редакції 23.06.2021

\section{REFERENCES}

1. Chirkov, O. Yu. (2021). Analysis of Models of Radiation Swelling and Radiation Creep, which take into account the Influence of Stresses, in the Problems of Mechanics of Inelastic Deformation. Part 1. Formulation of Defining Equations, Strength of Materials. 53, pp. 199-212. https://doi.org /10.1007/s11223-021-00276-0

2. Chirkov, O. Yu. (2021). Analysis of the Correctness of Problems in the Mechanics of Inelastic Deformation with the Influence of Stress State on the Processes of Radiation Swelling and Radiation Creep of the Material. Dopov. Nac. akad. nauk Ukr. 2021, 2, pp. 29 -37 (in Ukrainian). https://doi.org/10/15407/dopovidi2021.02.029

3. Chirkov, O. Yu. (2021). The Correctness of the Radiation Creep Equations that Take into Account Stress and Accumulated Irreversible Deformation in the Radiation Model Swelling of the Irradiated Material. Dopov. Nac. akad. nauk Ukr. 2021, 4, pp. 36-45 (in Ukrainian). https://doi.org/10.15407/dopovidi2021.04.036

4. Sorokin, A., Margolin, B. \& Kursevich, I. (2011). Effect of Neutron irradiation on mechanical properties of materials of WWER reactor internals. Iss. Mater. Sci., 2(66), pp. 131-151 (in Russian).

5. Margolin, B., Murashova, A. \& Neustroiev, V. (2012). Analysis of the Influence of Type Stress State on Radiation Swelling and Radiation Creep of Austenitic Steels, Strength of Materials, 44, pp. 227-240. https://doi.org/10.1007/s11223-012-9376-3

6. Ortega, J. M. \& Rheinboldt, W. C. (1970). Iterative Solution if Nonlinear Equations in Several Variables. New York; London: Academic Press.

Received 23.06.2021

O.Yu. Chirkov, https://orcid.org/0000-0003-1916-0277

Pisarenko Institute of Problems of Strength of the NAS of Ukraine, Kyiv

E-mail: chirkale82@gmail.com

\section{METHOD OF ELASTIC SOLUTIONS TO THE TASKS OF IRRADIATION CREEP CONSIDERING THE EFFECT \\ OF STRESSES AND ACCUMULATED IRREVERSIBLE STRAIN ON THE MATERIAL IRRADIATION SWELLING}

The method of elastic solutions for the irradiation creep nonlinear boundary values, which allows one to describe non-isothermal processes of inelastic deformation considering the irradiation swelling and creep of the irradiated material, is considered. To model the processes of irradiation swelling and creep, modern approaches are used considering the damaging dose, irradiation temperature, the influence of the stress state, and the accumulated irreversible strain. A modified method of elastic solutions for solving the boundary problems of irradiation creep is investigated. It is considered that the development and investigation of the iterative method properties within the tasks of radiation creep are complicated by the fact that it is necessary to account for a rather strict restriction associated with the asymmetry of the operator relating the errors of the iterative process for two successive 
approximations to verify their convergence and accuracy. Under such conditions, the standard approach of investigating the convergence of an iterative process concerning the properties of self-corrected operators is unacceptable. Moreover, the standard procedure of symmetrization of the equation for successive approximations leads to excessively conservative estimates of the convergence of the iterative method. Therefore, the optimization of its convergence rate has a rather approximate character. This problem is solved using a special regulatory requirement to analyze the convergence of successive approximations, which made it possible to develop a modified iterative process and to bring its local convergence to the general case of the equations of radiation creep. The modified process properties have been studied in detail. Based on the obtained results, the prior estimation of the asymptotic convergence rate of successive approximates has been obtained. The approaches to the optimization of the method of elastic solution regarding the tasks of irradiation creep are obtained.

Keywords: inelastic deformation, irradiation swelling, irradiation creep, method of elastic solutions, iteration process, convergence and accuracy of successive approximations. 